\title{
Experimental Evaluation of YouTube Performance on MPTCP-based LTE-WLAN Integration
}

\author{
Shashwat Kumar, Himank Gupta, Antony Franklin A. \\ Department of Computer Science and Engineering \\ Indian Institute of Technology Hyderabad, India \\ Email: \{cs15resch11011, cs16mtech01001, antony.franklin\}@iith.ac.in
}

\begin{abstract}
Soaring demand for high-quality and data-intensive services such as video streaming pushing the limits of the cellular networks. In due time, the bandwidth requirement for these services will exceed the cellular network capacity. Now, it is vital to aggregate cellular (licensed) and WLAN (unlicensed) bands to keep pace with the increasing high bandwidth requirement. Multipath TCP (MPTCP) is a transport layer protocol which can be used to aggregate available bandwidths of multiple networks at a multi-homed User Equipment (UE). We evaluate the performance of LTE-WLAN Integration(LWI) using MPTCP in terms of Quality of Experience (QoE) of YouTube Ultra-High Definition (UHD) videos in a test-bed. As YouTube server does not support MPTCP, we use an architecture with MPTCP proxy which enables the use of MPTCP services to multi-homed UEs. To analyse the QoE, we have developed an application to search and play the YouTube videos. This application monitors the key performance metrics to evaluate QoE in terms of Mean Opinion Score (MOS). Our results show significant improvement in MOS with LWI compared to WLAN or LTE alone. Also, our results indicate that more data is offloaded through Wi-Fi when using lowest RTT (Round Trip Time) scheduler in MPTCP.
\end{abstract}

\section{INTRODUCTION}

The latest market research conducted by Cisco indicates that video traffic accounts for 55 percent of the total mobile data usage over the Internet in 2014 and will reach 72 percent by the year 2019 [1]. Mobile data is expected to increase 10-fold globally from 2014 to 2019. As the throughput demand of high-quality video streaming outpaces the available capacity of cellular networks, it is important to exploit the multihoming feature of User Equipment (UEs) such as smartphones to integrate different networks technologies. UE should be able to use multiple available network interfaces simultaneously to achieve higher data rates. Many architectures have been proposed for interworking between LTE and WLAN by 3GPP from Release 8 [2]. Currently, 3GPP is working on standardising Radio Access Network (RAN) level LTEWLAN Aggregation (LWA) at PDCP layer in the LTE protocol stack [3] with two possible traffic steering mechanism, namely switched bearer and split bearer. In switched bearer, all the flows belong to a bearer is moved to Wi-Fi. However, in split bearer, a given bearer uses both LTE and Wi-Fi. The main issue with LWA is that the amount of data to be sent over LTE and Wi-Fi needs to be controlled by the eNB to utilise both network resources efficiently. It is still an open problem to find the optimal split for a given radio condition. The problem becomes even harder in the TCP scenario.
MPTCP is a promising transport-layer protocol that supports simultaneous use of multiple network interfaces at UE. MPTCP defines a set of extensions over the TCP, which allows the UEs to use multiple network interfaces for a single end-toend connection by creating multiple TCP sub-flows at sender side and merging sub-flows at the receiver side to create a single TCP stream for upper layer protocol [4]. Each TCP sub-flow could take different paths in the network to reach the destination. MPTCP is very helpful in mitigating network limitations, and it is also compatible with the TCP. MPTCP operates at the transport layer and aims to be transparent to both higher and lower layers. So MPTCP is used as the solution to integrate LTE and Wi-Fi in the mobile network. MPTCP can be used without any changes in the LTE architecture, and it can support both collocated and non-collocated network scenarios. Also, MPTCP does not require any special interface between eNB and UE to offload data over Wi-Fi. In MPTCP based LTE-Wi-Fi integration data split between Wi-Fi and LTE networks is taken care by MPTCP scheduler based on RTT values on individual network.

In this paper, we evaluate the performance of LWI using MPTCP in a test-bed using UHD video streaming from YouTube. To the best of our knowledge, this is the first-time, MPTCP over LTE and WLAN network has been studied in a test-bed scenario. The main contributions of this paper are as given below.

- Evaluation of LWI using MPTCP proxy in a real test-bed using open source platform for LTE.

- Video QoE measurement for YouTube videos in a multihomed UE using a new web application.

- Performance evaluation of LWI using MPTCP for UHD videos in term of video QoE compared to LTE or Wi-Fi used alone.

The remainder of the paper is organised as follows. Section II provides the related work. Section III describes the feasibility of LWI by deploying MPTCP proxy. We examine the MPTCP for YouTube video with a real-time test-bed in Section IV. Section V explains the application which we have developed to monitor the KPIs for YouTube and the approach to calculate MOS from the KPIs. Section VI evaluates the performance of LWI using MPTCP for YouTube in terms of QoE and we conclude the paper in Section VII with our finding. We use both Wi-Fi and WLAN interchangeably to 
refer to 802.11 based network.

\section{RELATED WORK}

Over the last five years, multiple research efforts have been carried out to enable MPTCP at the user end. Partov et. al. [5] explored MPTCP performance by a trace-driven approach using a python tool to send and receive packets through the LTE and WLAN interfaces simultaneously. Using this tool, they analysed different scheduling schemes for MPTCP.

Chen et. al [6] have studied the performance of MPTCP into the wild, using two different network scenarios with LTE+Wi$\mathrm{Fi}$ and $3 \mathrm{G}+\mathrm{Wi}-\mathrm{Fi}$. Their analysis shows that how MPTCP benefits the user for downloading the files of various sizes from an MPTCP enabled remote server. To analyse end-toend MPTCP behaviour, they deployed MPTCP server in their campus premises and performed evaluation from the remote place. De Coninck et al. [7] and Viet-Hoang Tran et al. [8] have evaluated MPTCP using Android application and showed that how applications using both interfaces on smart-phone can split the data over LTE and Wi-Fi. As application content servers are not MPTCP enabled, they have deployed MPTCP enabled proxy over the cloud to provide MPTCP support to the application. However, due to the deployment of MPTCP proxy over the cloud, every user requests have to go through the cloud server, which introduces the unnecessary delay at the user end. Wu et. al. [9] have presented a Quality-Driven MultIpath TCP (ADMIT) scheme that integrates the utility maximisation based Forward Error Correction (FEC) coding and rate allocation for streaming high-quality mobile video with MPTCP in Heterogeneous wireless networks.

Deng et. al. [10] have studied how network path quality affects the QoE of HTTP video streaming. They measured corelation between network QoS and application QoS. They also identified that the re-buffering frequency as a major factor for Mean Opinion Score (MOS) variance. Gmez et. al. [11] developed an Android application for YouTube QoE evaluation for wireless terminals that carries out measurements of objective QoS parameters, which are then mapped onto subjective QoE in terms of MOS using a utility function. Similar application "YomoApp" have been developed by Wamser et. al. [12], which passively monitors key performance indicators (KPIs) of YouTube adaptive video streaming on a smartphone. They did not consider multi-homed mobile devices and performed the FLV parsing to find the KPIs. Mok et. al. [13] have characterised the correlation between the application and network QoS and performed subjective experiments to evaluate the relationship between application QoS and QoE. In their work, they concluded that the frequency of re-buffering is the main factor responsible for the variations in video $\mathrm{QoE}$.

All of these studies did not analyse the effect of LWI on video QoE. Moreover, they have studied the performance of MPTCP by deploying it locally on MPTCP enabled server or by measurement in the environment where all the traffic is controlled. In contrast, we study the performance of LWI for UHD videos on YouTube, with real WLAN and LTE testbed, focused on QoE factors, that better represent the real time MPTCP experience for the end users.

\section{LTE-WLAN INTEGRATION USING MPTCP}

In LWA, LTE Evolved NodeB (eNB) can schedule packets/flows to be served on LTE and Wi-Fi. 3GPP defined two possible scenarios for LWA: non-collocated where WLAN Access Point (AP) and eNB connects through a standardised $X_{w}$ interface and collocated in which Wi-Fi AP and LTE small cell are placed in the same box. This LWA requires changes in LTE architecture and UE and needs upgrading UEs and eNBs.

LWI can be achieved by using MPTCP at user and server equipment. Software changes are required at UE and content server to enable the end-to-end use of MPTCP. Some methods are available to allow the use of LWI without making changes on content servers as MPTCP is not deployed widely. So, if an UE has MPTCP enabled it can use LWI without any deployment dependency on end servers as shown in Fig. 1 below using MPTCP proxy.

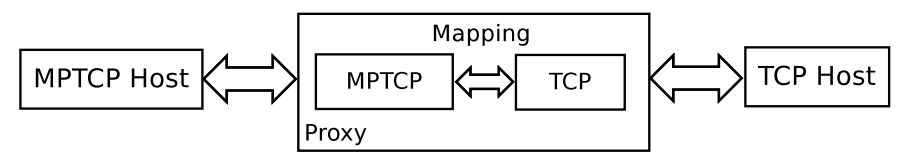

Fig. 1. MPTCP proxy mechanism.

Network operators, who wish to enable the LWI on their network, can deploy this proxy mechanism. Such mechanism has been considered by some mobile network operators [14]. MPTCP proxy accepts the MPTCP connection from MPTCP enabled clients and maps the sub-flows of the MPTCP connection to different TCP flow towards the end server.

Service quality can be analysed with QoS based on some measurement parameters such as data rate, but it does not account quality perceived by the user i.e. QoE which represents user experience. The $\mathrm{QoE}$ has usually been evaluated through subjective tests carried out by the users to assess the degree of satisfaction using MOS indicator. This type of approach is of course quite expensive, as well as annoying to the user. In recent years, new methods have been used to estimate the QoE based on certain performance indicators called Key Performance Indicators (KPIs). These indicators can be measured by the UE and help in QoE measurement. In this work, we evaluate LWI with MPTCP using Ultra High Definition (UHD) videos from YouTube. To enable the use of LWI, we use an architecture with MPTCP proxy at the edge of the LTE network towards the Internet as shown in Fig. 2. To measure the KPIs for the video, we have developed an application that captures the KPIs for the YouTube videos, i.e., initial load time, buffer time, and buffer frequency and these KPIs are used to calculate MOS [13].

To analyse the MPTCP behaviour, trace files have been generated at the end device and used to find the data split between the available paths. 


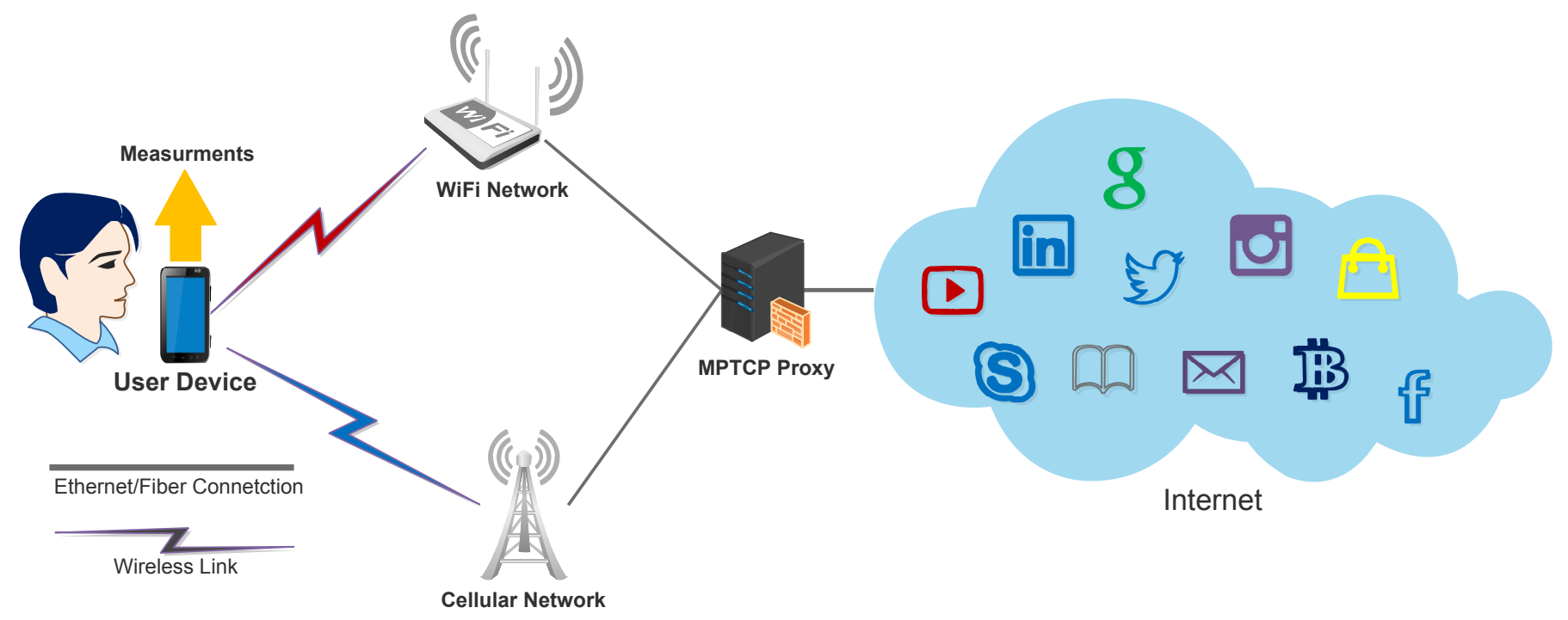

Fig. 2. System architecture to enable MPTCP with an MPTCP proxy server between UE and content server.

\section{TEST-Bed SETUP}

Fig. 2 illustrates the system architecture for LWI using an MPTCP proxy server. This architecture allows a user to use MPTCP service even though end server is not MPTCP enabled. If content severs is not MPTCP enabled proxy server converts MPTCP sub-flows to TCP flows otherwise it just forward the packets to the content server, allowing the user to use multiple network interface simultaneously to fetch the data from the server. Fig. 3 shows our test-bed setup used for experiments. For LTE network, we deployed OAI Software Alliance (OSA) test-bed which is developed by Eurecom [15]. OSA provides a standard-compliant implementation of a subset of Release 10 LTE for eNB, Mobility Management Entity (MME), Home Subscriber Server (HSS), Serving Gateway (SGW), and PDN Gateway (PGW) on standard Linux-based computing equipment (Intel x86 PC/ARM architectures). OAI LTE network has eNodeB (eNB) and Evolved Packet Core (EPC). In the test-bed, eNB, shown in Fig. 4, is deployed on a Linux machine with $3.4 \mathrm{GHz}$ Intel Core i7 processor and 8GB DDR3 RAM, running 3.19 low latency Linux kernel. USRP B210 is used as LTE RF transmitter/receiver with 25 resource blocks, connected via USB 3.0 to an eNodeB system. MPTCP proxy is deployed on a machine with $2.4 \mathrm{GHz}$ Intel Core i3 processor and 4GB DDR3 RAM, running on 3.19 MPTCP Linux kernel with MPTCP version 0.9 and SQUID proxy version 3.1 [16]. We disable the caching SQUID proxy to avoid performance improvement due to cached data on the proxy server. MPTCP proxy works as a converter between MPTCP and TCP connections. UE creates the MPTCP subflows to connect with the proxy, and as the content server is not MPTCP enabled, MPTCP proxy creates separate TCP flow towards YouTube server for each sub-flow. We use a commercial Google Nexus 5 as LTE device running rooted Android 4.0 with MPTCP patch [17]. We use a programmable sim card with predefined network parameters (such as IMEI
No., IMSI No., and MSISDN No.) to connect the Nexus 5 with the OAI testbed over LTE band 7. We use LTE band seven with 25 resource blocks as current version of OAI provide stable connection for these testbed settings. We use campus WLAN AP for Wi-Fi connectivity. Many users are attached to the AP and load varies with time as users join and leave the network. Several interfering WLAN networks are also operating. WiFi download speed varies according to traffic load and some users connected to the network. To generate and analyse the packet trace, we used Wireshark on the end device which is a mobile workstation. This mobile workstation is connected to LTE network through USB Tethering with Nexus 5 and to WLAN through a Wi-Fi dongle. Traces generated during the experiment are used to find how data is split between LTE and Wi-Fi.

\section{Video QoE Measurement using Web Application}

We have developed a hybrid application using IONIC framework to monitor the KPIs for YouTube videos to calculate the QoE in terms of MOS. The functionality of the application is made similar to YouTube using its data and player APIs. This application also collects the following KPIs to calculate the MOS of the video being played.

- Load/Initial Buffer Time $\left(T_{\text {init }}\right)$ : time taken by the YouTube player to buffer initial data before starting playback.

- Re-buffering Frequency $\left(f_{\text {rebuf }}\right)$ : frequency of rebuffering events during the playback.

- Mean re-buffering time $\left(T_{\text {rebuf }}\right)$ : average duration of a re-buffering event.

Each of these performance metrics $\left(T_{\text {init }}, f_{\text {rebuf }}\right.$, and $\left.T_{\text {rebuf }}\right)$ is divided into three levels (low, medium, and high) which are based on the $25^{t h}, 50^{t h}$, and $75^{t h}$ percentiles of the actual metric [13]. The quantified performance metrics $L_{t i}, L_{f r}$, and $L_{t r}$ take the numerical values 1,2 , and 3 to represent 


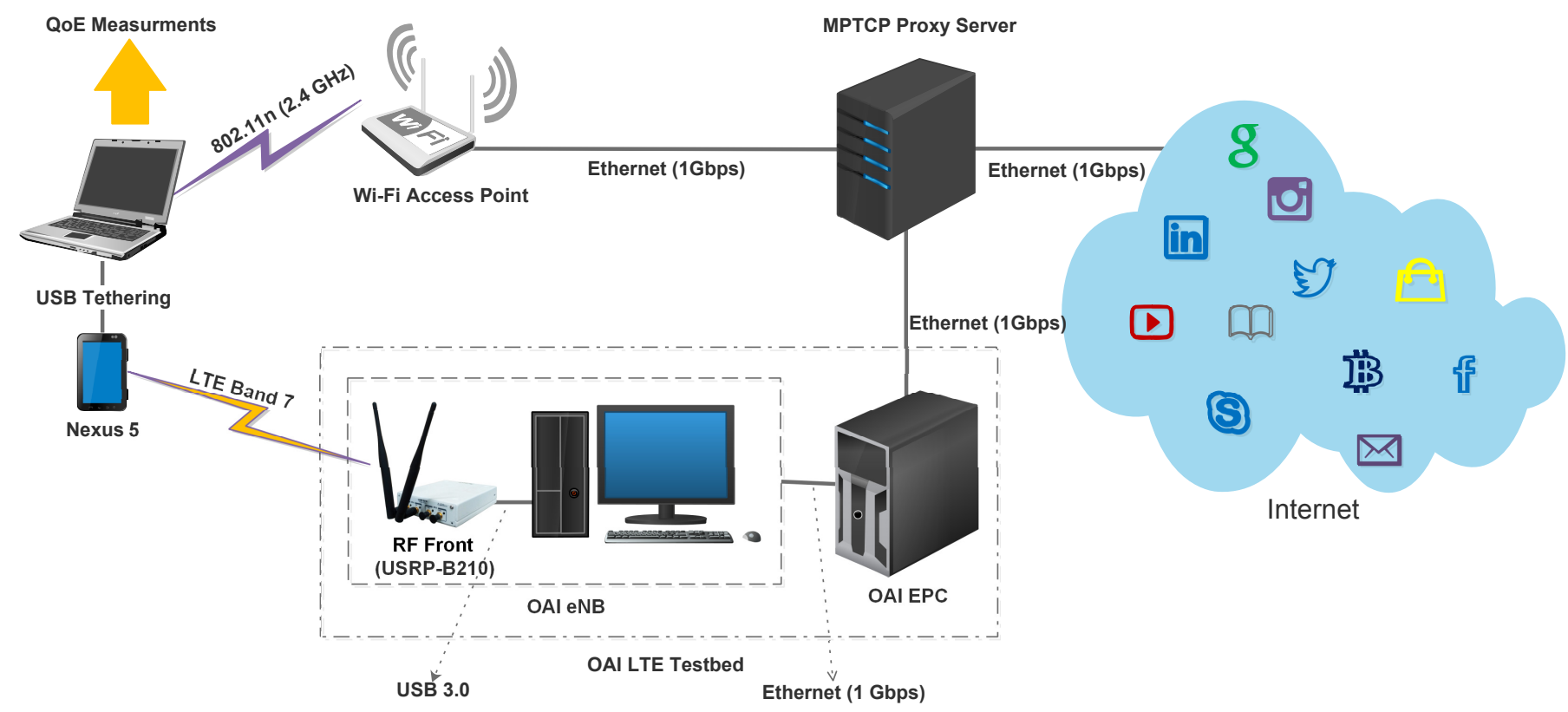

Fig. 3. OAI LTE test-bed setup showing LTE eNB connected with USRP B210 RF front, EPC, MPTCP proxy, and UE.

TABLE I

THREE VALUED LEVELS OF APPLICATION PERFORMANCE METRICS

\begin{tabular}{|c|c|c|c|c|c|}
\hline$T_{\text {init }}$ & $L_{t i}$ & $f_{\text {rebuf }}$ & $L_{f r}$ & $T_{\text {rebuf }}$ & $L_{t r}$ \\
\hline 0 to $1 \mathrm{Sec}$ & 1 & 0 to 0.02 & 1 & 0 to $5 \mathrm{Sec}$ & 1 \\
\hline 1 to $5 \mathrm{Sec}$ & 2 & 0.02 to 0.15 & 2 & 5 to $10 \mathrm{Sec}$ & 2 \\
\hline$>5 \mathrm{Sec}$ & 3 & $>0.15$ & 3 & $>10 \mathrm{Sec}$ & 3 \\
\hline
\end{tabular}

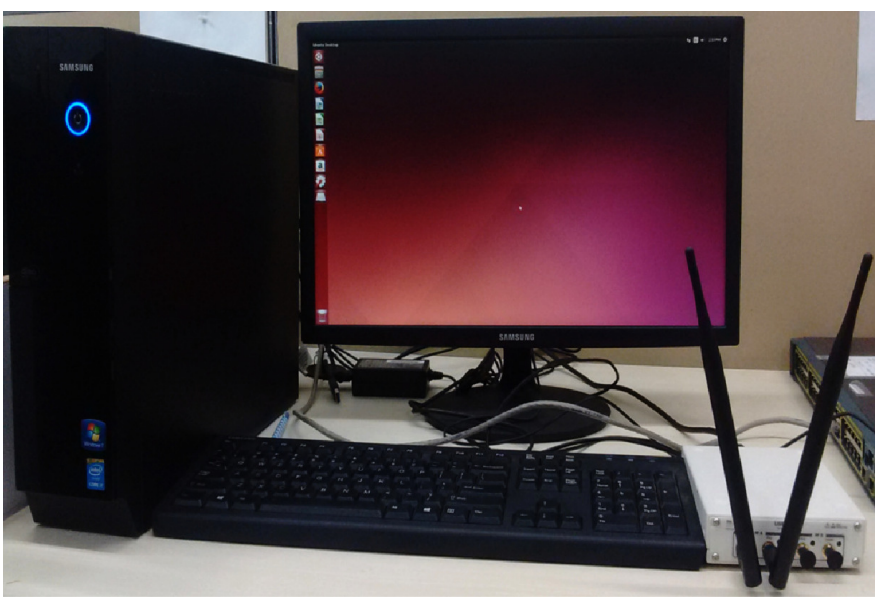

Fig. 4. OAI eNB connected to USRP B210 board (RF Front) via USB 3.0 in OAI LTE test-bed setup.

low, medium, and high levels, respectively, The mapping from $T_{\text {init }}, f_{\text {rebuf }}$, and $T_{\text {rebuf }}$ to $L_{t i}, L_{f r}$, and $L_{t r}$ is done according to Table I [13]. The quantified performance metrics from Table I is used in [13] to obtain the MOS estimation from linear regression as given below.

$$
\text { MOS }=4.23-0.0672 \times L_{t i}-0.742 \times L_{f r}-0.106 \times L_{t r}
$$

From the Eq. 1, it is clear that the maximum value of MOS can be 3.3148 for minimum value of $L_{t i}, L_{f r}$, and $L_{t r}$ from Table I. Re-buffering frequency metric $\left(L_{f r}\right)$ has a high impact on QoE compared to initial buffering time $\left(L_{\text {init }}\right)$ and rebuffering duration $\left(L_{t r}\right)$. As YouTube server is not MPTCP enabled, we deployed MPTCP proxy and configured the UE to use this proxy server. To analyse the behaviour of MPTCP with YouTube, we generated network traces on the end device and analysed them to measure the packet split done by MPTCP.

TABLE II

RTT (MS) BETWEEN UE TO MPTCP PROXY THROUGH LTE AND WI-FI NETWORKS AND MPTCP PROXY TO YOUTUBE SERVER

\begin{tabular}{|c|c|c|c|c|}
\hline & Min & Avg & Max & Mdev \\
\hline LTE & 12.052 & 17.51 & 26.19 & 3.87 \\
\hline Wi-Fi & 2.67 & 11.686 & 30.562 & 6.893 \\
\hline MPTCP Proxy & 24.613 & 24.751 & 24.945 & 0.129 \\
\hline
\end{tabular}

\section{Results AND ANALYsis}

Sub-flow RTT is imperative for LWI performance as MPTCP default scheduler "Lowest RTT" first sends data over sub-flow with lowest RTT until its congestion window is full, then it starts transmitting on the sub-flow with the next higher RTT. "Round Robin" another scheduler in MPTCP, send equal data over each sub-flow regardless of the properties of link used by sub-flow and "Redundant" scheduler yield data redundancy to achieve better success rate which may batter the performance of LWI as some of the data being sent over multiple channels will result as inefficient channel utilization. In our experiments, we use default "Lowest RTT" scheduler. As lowest RTT first scheduler depends on the RTT of the subflows so we measure the RTT of different paths to analyse the path priority of scheduler. Table II shows the RTT in 


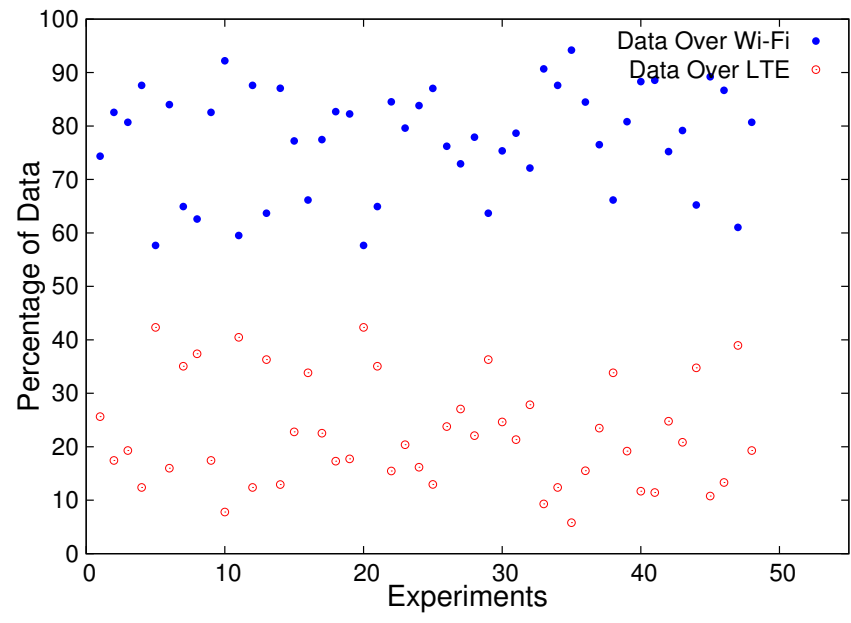

Fig. 5. Percentage of data sent over Wi-Fi and LTE for LWI

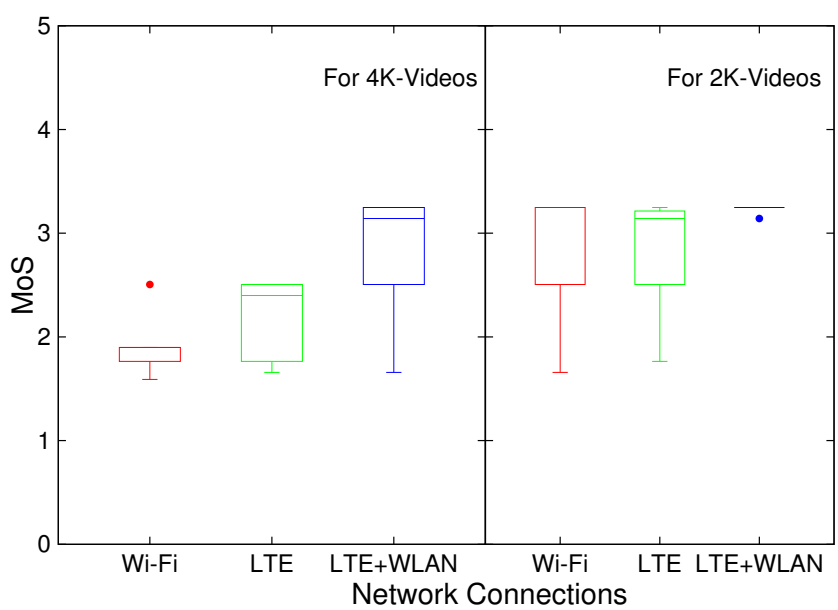

Fig. 6. Value of MOS for LWI, LTE, and Wi-Fi for $4 \mathrm{~K}$ and $2 \mathrm{~K}$ videos.

milliseconds (ms) from the UE to MPTCP proxy while using LTE and Wi-Fi.

Wi-Fi is used to establish primary sub-flow which carries the MP_CAPABLE and other control option of MPTCP to the server. It is evident from the data in the table II that Wi-Fi has lower RTT than LTE. Fig. 5 represents data split for different experiments between LTE and Wi-Fi networks for LWI. The plot shows that MPTCP sends more data over Wi-Fi network which is justified as Wi-Fi has lower RTT than LTE and also used as primary sub-flow. MPTCP path scheduler split the data based on the sub-flow RTT, so user or application cannot control the data split (for the current version of MPTCP) among the different paths in LWI using MPTCP, and it may also impact the LWI performance if sub-flow with lowest RTT is over a network with poor channel quality. For our video QoE experiments, we use "Samsung Galaxy S6 4K Ultra HD Video Sample" [18] which has a playtime of 66 seconds and download size of $131 \mathrm{MB}$ for $4 \mathrm{~K}(3840 \mathrm{p}$ $\mathrm{x} 2160 \mathrm{p})$ and $50 \mathrm{MB}$ for $2 \mathrm{~K}(2560 \mathrm{p}$ x 1440p) resolutions. We calculate the MOS using Eq. 1 with quantized KIPs from

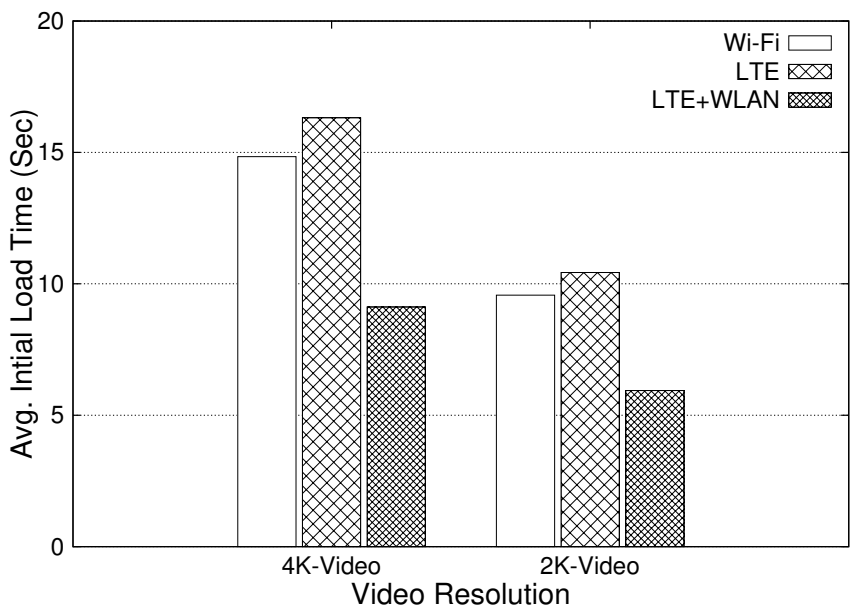

Fig. 7. Average initial load time for $4 \mathrm{~K}$ and $2 \mathrm{~K}$ videos using LWI, LTE, and Wi-Fi.

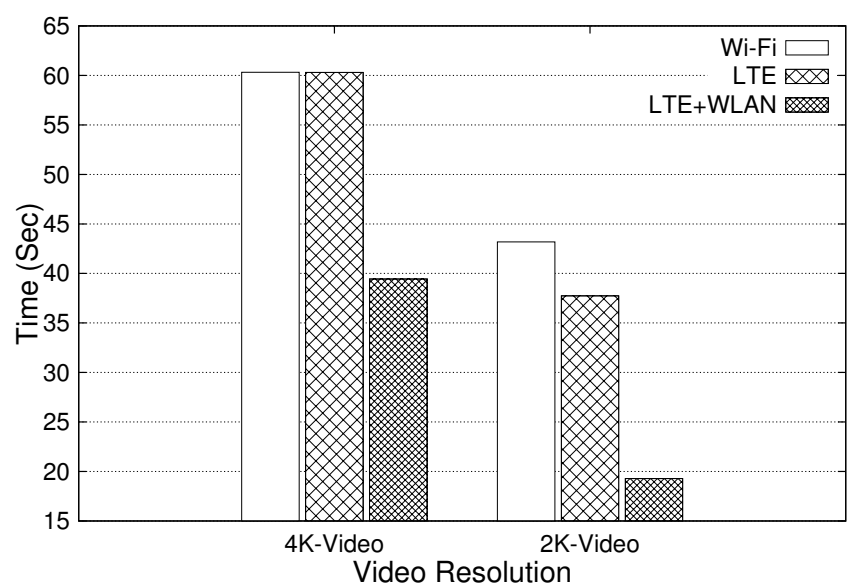

Fig. 8. Average time taken to buffer the complete video.

Table I using the data collected from the experiments. Fig. 6 shows the MOS for $4 \mathrm{~K}$ and $2 \mathrm{~K}$ videos for LTE, Wi-Fi, and LWI. For $4 \mathrm{~K}$ videos, LWI shows momentous improvement over single TCP connection using LTE and Wi-Fi. LWI has $34.4 \%$ better MOS value compared to LTE and $20.4 \%$ better than Wi-Fi for $4 \mathrm{~K}$ videos. For $2 \mathrm{~K}$ videos, network integration shows minimal improvement in MOS value over single TCP with LTE and $\mathrm{Wi}-\mathrm{Fi}$ as the bit rate for YouTube $2 \mathrm{~K}$ video for standard frame rate (30fps) is $8.4 \mathrm{Mbps}$ which is nearly equal to the bandwidth available in both LTE and Wi-Fi networks. Over MPTCP, there is no variation on MOS as the available datarate is significantly increased by using LWI. Also, the data is split over LTE and Wi-Fi to reduce the data usage over LTE. The user need not worry about which network to use after seeing some degradation in the video playback.

Fig. 7 shows initial load time for $4 \mathrm{~K}$ and $2 \mathrm{~K}$ video in seconds using Wi-Fi, LTE, and LWI. Initial load time consists of connection setup time and time to buffer the required data before a player can start playing the video. In this phase, 
TABLE III

AVERAGE NUMBER OF REBUFFERINGS $\left(N_{\text {rebuf }}\right.$ ) AND AVERAGE TIME TAKEN IN REBUFFERING $\left(T_{\text {rebuf }}\right.$ ) FOR LTE, WI-FI, AND LTE-WLAN INTEGRATIOPN FOR 4K AND $2 \mathrm{~K}$ VIDEOS

\begin{tabular}{|l|l|l|l|l|}
\hline & \multicolumn{2}{|c|}{$4 \mathrm{~K}$} & \multicolumn{2}{c|}{$2 \mathrm{~K}$} \\
\hline & $N_{\text {rebuf }}$ & $T_{\text {rebuf }}(\mathrm{Sec})$. & $N_{\text {rebuf }}$ & $T_{\text {rebuf }}(\mathrm{Sec})$. \\
\hline Wi-Fi & 5.32 & 4.55 & 3.67 & 2.75 \\
\hline LTE & 6.44 & 5.89 & 4.26 & 3.83 \\
\hline LTE-WLAN & 2.38 & 3.69 & 0.88 & 2.01 \\
\hline
\end{tabular}

YouTube server send the meta-data regarding the video to the application, on receiving meta-data application send the request for the $4 \mathrm{~K} / 2 \mathrm{~K}$ video to the server and start buffering the data sent by the server to start playing video.

MPTCP sends one DUPACK over the primary sub-flow to add a new sub-flow in existing connection which takes relatively very less time compared to initial load time. After adding a new sub-flow, both sub-flows can be used to buffer the initial data, so LWI shows the improvement for initial load time. By the use of LWI, initial load time is reduced by $35.0 \%$ and $43.0 \%$ compared to $\mathrm{Wi}-\mathrm{Fi}$ and LTE respectively.

Fig. 8 shows time to buffer complete video with LTE, Wi-Fi and LWI. Here, MPTCP having a significant advantage over LTE and Wi-Fi network. This dominance feature of LTE-Wi$\mathrm{Fi}$ integration for $4 \mathrm{~K}$ videos does have a convincing impact on YouTube offline feature where the user gets YouTube video offline to watch later. Table III represents the data regarding the average number of buffering events and average buffer duration experienced by the user during playtime of the video. Results evidently show that LWI provides better performance than LTE and Wi-Fi alone. The number of average buffering events and average buffer duration is reduced significantly.

\section{CONCLUSION AND Future WORK}

LWI is necessary to cater the high quality and data intensive services with acceptable user QoE. MPTCP can be used to facilitate the LWI. As MPTCP requires the modification of end server and user equipment to provide end-to-end MPTCP connection, MPTCP proxy can be employed in the operator's network to enable MPTCP based LWI. We evaluated the performance of MPTCP based LWI architecture using a realtime LTE testbed and Wi-Fi for UHD videos. Results show that the MOS can be increased up to $34.4 \%$ compared to LTE and $20.4 \%$ as compared to $\mathrm{Wi}-\mathrm{Fi}$ alone for $4 \mathrm{~K}$ videos. The probability of the user seeing a video depends on the initial load time, and MPTCP can reduce initial load time by $17.39 \%$ by using MPTCP. Results show that more data is sent over Wi-Fi compared to LTE as WLAN has less RTT than LTE in our experiments. Evaluated architecture is easy to deploy, and any network operator who wishes to provide LWI without changing the LTE architecture can deploy it with small capital expenditure. The operator should deploy Wi-Fi with LTE to ensure QoE improvement as the use of networks with heterogeneous QoS may reduce the MPTCP performance. As part of our future work, we plan to study how to control the packet split over LTE and Wi-Fi to offload more traffic over Wi-Fi without much degradation in video QoE.

\section{REFERENCES}

[1] Cisco, "Cisco Visual Networking Index : Forecast and Methodology , 2013-2018," 2014.

[2] Qualcomm Inc., Intel Corporation, and China Telecom, "LTE-WLAN Radio Level Integration and Interworking Enhancement," 2015. [Online]. Available: www.3gpp.org/ftp/meetings_3gpp_sync/ran/Inbox/RP150510.zip

[3] QualComm, "Motivation for LTE-WiFi Aggregation," 2015. [Online]. Available: Available: http://www.3gpp.org/ftp/tsg_ran/TSG_RAN/TSGR_64/Docs/RP140739.zip

[4] A. Ford, C. Raiciu, M. Handley, S. Barre, and J. Iyengar, "Architectural guidelines for multipath tcp development," Internet Requests for Comments, IETF, RFC 6182, March 2011. [Online]. Available: https://tools.ietf.org/html/rfc6182

[5] B. Partov and D. J. Leith, "Experimental evaluation of Multi-path schedulers for 1te/Wi-Fi Devices," in Proceedings of the Tenth ACM International Workshop on Wireless Network Testbeds, Experimental Evaluation, and Characterization, ser. WiNTECH'16. New York, NY, USA: ACM, 2016, pp. 41-48.

[6] Y.-C. Chen, Y.-s. Lim, R. J. Gibbens, E. M. Nahum, R. Khalili, and D. Towsley, "A Measurement-based Study of MultiPath TCP Performance over Wireless Networks," in Proceedings of the 2013 Conference on Internet Measurement Conference, ser. IMC '13. ACM, 2013, pp. $455-468$.

[7] Q. De Coninck, M. Baerts, B. Hesmans, and O. Bonaventure, "Poster: Evaluating android applications with multipath tcp," in Proceedings of the 21st Annual International Conference on Mobile Computing and Networking, ser. MobiCom '15. New York, NY, USA: ACM, 2015, pp. 230-232.

[8] V.-H. Tran, Q. D. Coninck, B. Hesmans, R. Sadre, and O. Bonaventure, "Observing real Multipath TCP traffic," Computer Communications, vol. 94, pp. $114-122,2016$

[9] J. Wu, C. Yuen, B. Cheng, M. Wang, and J. Chen, "Streaming HighQuality Mobile Video with Multipath TCP in Heterogeneous Wireless Networks," IEEE Transactions on Mobile Computing, vol. 15, no. 9, pp. 2345-2361, 2016

[10] S. Deng, R. Netravali, A. Sivaraman, and H. Balakrishnan, "WiFi, LTE, or Both?: Measuring Multi-Homed Wireless Internet Performance," in Proceedings of the 2014 Conference on Internet Measurement Conference, ser. IMC '14. New York, NY, USA: ACM, 2014, pp. 181-194.

[11] G. Gómez, L. Hortigüela, Q. Pérez, J. Lorca, R. García, and M. C. Aguayo-Torres, "YouTube QoE evaluation tool for Android wireless terminals," EURASIP Journal on Wireless Communications and Networking, vol. 2014, no. 1, p. 164, 2014.

[12] F. Wamser, M. Seufert, P. Casas, R. Irmer, P. Tran-Gia, and R. Schatz "YoMoApp: A tool for analyzing QoE of YouTube HTTP adaptive streaming in mobile networks," in Networks and Communications (EuCNC), 2015 European Conference on, June 2015, pp. 239-243.

[13] R. K. P. Mok, E. W. W. Chan, and R. K. C. Chang, "Measuring the quality of experience of HTTP video streaming," in 12th IFIP/IEEE International Symposium on Integrated Network Management (IM 2011) and Workshops, 2011, pp. 485-492.

[14] S. SungHoon, J. Ryu, J. Kim, S. Min, C. Oh, and H. J, "KT's MPTCP Proxy Experiences - Deployment and testing considerations," IETF 91 MPTCP WG, Tech. Rep., 2014. [Online]. Available: https://www.ietf.org/proceedings/91/slides/slides-91-mptcp-5.pdf

[15] "OpenAirInterface - 5G software alliance for democratising wireless innovation," http://www.openairinterface.org/.

[16] “Squid: Optimising web delivery," http://www.squid-cache.org/.

[17] "MultiPath TCP - linux kernel implementation," http://multipathtcp.org/.

[18] "Samsung Galaxy S6 4K Ultra HD Video Sample," https://www.youtube.com/watch?v=onXpKXbnbE0, accessed: 2016-1025. 